\title{
I ndication and validity of ultrasonographic evaluation for assessment of vocal fold mobility before and after thyroid surgery
}

\author{
Aya Ebina ${ }^{1}$, I wao Sugitani ${ }^{2}$, Yorihisa Orita $^{3}$, Soshi Takao ${ }^{4}$, Kiyoaki Tukahara $^{5}, K^{2}$ iko Yamada ${ }^{6}$, \\ Kazuyoshi Kawabata ${ }^{1}$
}

1. Division of Head and Neck, Cancer Institute Hospital, Japanese Foundation for Cancer Research, Tokyo, Japan. 2. Division of Endocrine Surgery, Department of Surgery, Nippon Medical School, Tokyo, Japan. 3. Department of Otolaryngology Head and Neck Surgery, Okayama University Graduate School of Medicine, Dentistry and Pharmaceutical Sciences, Okayama, Japan. 4. Department of Epidemiology, Okayama University Graduate School of Medicine, Dentistry and Pharmaceutical Sciences, Okayama, Japan. 5. Department of Otolaryngology Head and Neck Surgery, Tokyo Medical University Hachioji Medical Center, Tokyo, Japan. 6. Division of Ultrasonography, Cancer Institute Hospital, Japanese Foundation for Cancer Research, Tokyo, Japan

Correspondence: Aya Ebina. Address: Division of Head and Neck, Cancer Institute Hospital, 3-8-31 Ariake, Koto-ku, Tokyo 135-8550, Japan. Email: aya.ebina@jfcr.or.jp

Received: August 23, 2015

DOI : $10.5430 /$ ijdi.v3n1p26
Accepted: October 19, 2015

URL: http://dx.doi.org/10.5430/ijdi.v3n1p26

\section{Abstract}

Background: Evaluating recurrent laryngeal nerve function before and after thyroid surgery is important. Although laryngeal electroendoscope is the most reliable tool for evaluating vocal fold (VF) mobility, ultrasonography (US) may offer a non-invasive alternative. The aim of this study is to evaluate the validity and limitation of US evaluation for the assessment of VF mobility.

Methods: This was a study for diagnostic testing of US in a single center. One hundred and one patients who underwent thyroid surgery between February 2011 and August 2011 were enrolled to this study. Observation of the VF mobility using a B-mode US scanner was performed before and after thyroid surgery, through the thyrohyoid membrane, thyroid notch, and cricothyroid membrane. The results of US were compared with those of electroendoscopic examination which was performed on the same day but after the US.

Results: First we excluded one case that could not be observed by electroendoscopic examination before surgery. After excluding the cases which the VF mobility was invisible by US, we analyzed 89 out of the 100 patients before thyroid surgery and 89 out of the 101 patients after surgery. The sensitivity and specificity of US were $87.5 \%, 100 \%$ for before and $94.4 \%, 100 \%$ for after surgery, respectively. It was significantly more difficult to assess the VF mobility by US among patients who were men (odds ratio [OR] 24.7, 95\% confidence interval [CI] 4.9-124.2), 65 years old or more (OR 3.5, 95\% CI 1.0-11.9), and have past history of thyroid surgery (OR 7.9, 95\% CI 2.1-29.2). Being obese was also a significant factor (OR 10.5, 95\% CI 1.7-64.8) among younger patients (less than 65 years).

Conclusion: US evaluation can be used for the assessment of recurrent laryngeal nerve function before and after thyroid surgery. This is a safe and non-invasive diagnostic tool which may be performed as a first-line method.

\section{Keywords}

Ultrasonography, Thyroid surgery, Recurrent laryngeal nerve, Vocal fold 


\section{I ntroduction}

Functional evaluation of the recurrent laryngeal nerve before and after thyroid surgery is very important. First, preoperative recurrent laryngeal nerve palsy may indicate extrathyroidal invasion of the tumor which may suggest poor prognosis of the patients ${ }^{[1]}$. The proper risk definition of the tumor results in appropriate selective approach to patient treatment and postoperative follow-up. Second, if recurrent laryngeal nerve palsy is identified before surgery, the surgeon can be prepared in case that an accidental injury of the contralateral healthy side occurs, and early detection of need for tracheostomy will prevent the patients from suffocation ${ }^{[2-5]}$. At last, since recurrent laryngeal nerve palsy is frequent complication occurring in thyroid surgery, absence of postoperative recurrent laryngeal nerve palsy can be one of the criteria for proper surgical quality ${ }^{[6,7]}$. Laryngeal fiberscope, now electroendoscope is in popular use in Japan, is the most common and reliable instrument for directly checking vocal fold (VF) mobility, but is a little invasive and uncomfortable for patients ${ }^{[2,8]}$. In addition, a surgeon who undergoes thyroid surgery is not always proficient in handling a laryngeal fiberscope. Currently, recurrent laryngeal nerve function is also evaluated using the other non-invasive methods such as ultrasonography (US) or electromyography, and especially US could evaluate VF mobility in over $85 \%$ of patients with thyroid disease ${ }^{[6,9,10]}$. Now US evaluation is almost indispensable to the patients with thyroid disease, and all we have to do is properly placing the US probe above the thyroid cartilage to observe the VF mobility after routine examination for thyroid and neck lymph nodes ${ }^{[6]}$. Although US can't detect subtle morphologic abnormalities of the larynx such as laryngeal carcinoma in the first stage of the disease, it may be a useful and cost-effective screening tool for observation of VF mobility. The aim of the present study was to investigate the feasibility and validity of US evaluation for assessment of VF mobility before and after thyroid surgery, and elucidate the factors which make it difficult for US to assess the VF mobility.

\section{Matients and methods}

\subsection{Eligibility criteria for participants}

Patients who underwent thyroid surgery at Cancer Institute Hospital, tertiary oncology referral center in Japan, between February 2011 and August 2011, were considered as candidates for inclusion in the study. All patients were Japanese and provided written informed consent to participate. All thyroid surgeries were performed by conventional technique with subplatysmal skin flaps, and electocautery was used to make incisions and create skin flaps. Patients who had undergone laryngeal surgery (e.g. partial or total laryngectomy) were not included to the study. All study protocols have been reviewed and approved by the institutional review board.

\subsection{Observation of the vocal fold}

All eligible patients underwent both of the electroendoscopic examination and neck US evaluation on the same days, the days before and after the thyroid surgery. Neck US evaluations (HITACHI-ALOCA, Tokyo, Japan) were performed using 7-18-MHz linear transducer by an experienced radiologist (K.Y), with special regard to VF mobility. During the US examination, the patient was placed in a supine position with the neck slightly extended and a pad under the shoulders to provide optimum exposure of the neck. First we asked all the patients if they had any neck problems like cervical spondylosis, and when they had some neck problems they were positioned at normal supine position. After our routine observation of the thyroid gland and neck lymph nodes, VF evaluation was performed. The US probe was placed transversely along the midline of the neck at the following three points: 1) the thyrohyoid membrane; 2) the thyroid notch; and 3) the cricothyroid membrane (see Figure 1). After the US evaluations, electroendoscopic examinations (OLYMPUS, Tokyo, Japan) via the nasal route were performed while having the patient in a seated position by an experienced otolaryngologist (A. E). 


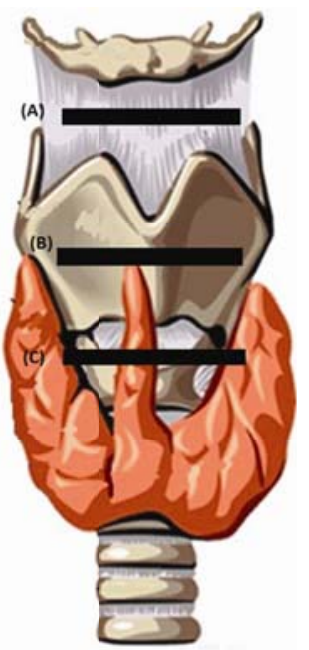

Figure 1. The 3 points to place the transducer of ultrasonography to observe the vocal fold
The 3 points to place the transducer of ultrasonography to observe the vocal fold. The transducer was placed transversely over three points: A) thyrohyoid membrane; B) thyroid notch; and C) cricothyroid membrane. The black bars indicate the location of the transducer.

\subsection{Outcome assessment}

In the US evaluation, false VFs appear as hyperechoic structures while true VFs are hypoechoic, and the vocal ligament (free edge of the true cord) is seen as an echogenic band ${ }^{[11]}$. Normal recurrent laryngeal nerve function was defined as symmetrically abductive and adductive motions of the true and false VFs during deep respiration and phonation. Paralyzed recurrent laryngeal nerve was defined as limited motion of those movements or paradoxical movement (see Figure 2). There was one rare case whose VFs were covered by a protruding metastatic retropharyngeal lymph node and could not be fully observed by electroendoscopic examination before thyroid surgery, and we excluded this case from the analysis. There were no cases whose VFs could not be observed by electroendoscopic examination after surgeries. Any other abnormalities of the larynx than

paralysis of recurrent laryngeal nerve (e.g. laryngeal tumor) have never been found by erectroendoscopic examination among the 101 patients. We compared the results of US evaluation with those of electroendoscopic examination among cases of which VFs could be visualized by US. Since observation for right and left VF was considered to be essentially independent, we combined these observations.

\subsection{Statistical analysis}

We estimated sensitivity, specificity, positive and negative predictive values to evaluate validity of US evaluation compared with electroendoscopic examination. Then, we calculated crude odds ratios (ORs) and $95 \%$ confidence intervals (CIs) to examine the association between patients' profiles (sex, age, obesity, and prior operation history of thyroid) and application of US evaluation (NOT being able to visualize $v s$. being able to visualize). Ages were dichotomized into less than 65 years old and 65 years old or more. Obesity was defined by body mass index (BMI) (more than or equal to 25). In addition, we calculated stratum specific ORs with obesity stratified by sex, age, and prior surgical history instead of logistic regression analysis for adjusting those factors at the same time. The reason why we employed stratified analysis was that the influences of factors except obesity was obvious regardless of other factors and adjusted ORs did not converge due to small numbers (actually, zero) of some cells. $P$ values of less than 0.05 (two-sided test) were considered statistically significant. All analyses were performed using STATA 12.1 (StataCorp, College Station, TX, USA) and JMP version 11 (SAS Institute Inc., Cary, NC USA).

\section{Results}

Table 1 describes the characteristics of the study patients of which $75 \%$ were women. Mean age at the time of thyroid surgery was 54 years (range, 18-78 years). The age distributions were similar between women and men. Of the 101 patients, 92 (91\%) patients had a surgery for papillary thyroid carcinoma, $3(3 \%)$ patients for anaplastic thyroid carcinoma, 2 (2\%) patients for medullary thyroid carcinoma, 1 (1\%) patient for follicular thyroid carcinoma, and the remaining $3(3 \%)$ patients for benign thyroid tumors. As for surgical procedure, 55 (54\%) patients underwent hemithyroidectomy, 30 (30\%) patients had total thyroidectomy, and $16(16 \%)$ patients had only neck dissection. All of these 16 patients who underwent only neck dissection had experienced prior thyroid surgeries. 
Table 1. Characteristics of the 101 patients who underwent thyroid surgery at Cancer Institute Hospital, Japan, 2011 $(\mathrm{n}=101)$

\begin{tabular}{|c|c|}
\hline Characteristics & No. of patients (\%) \\
\hline \multicolumn{2}{|l|}{ Sex } \\
\hline Female & $76(75 \%)$ \\
\hline Male & $25(25 \%)$ \\
\hline Age (years), mean & 54 (range, $17-78$ years) \\
\hline Female & $53 \pm 30$ \\
\hline Male & $53 \pm 25$ \\
\hline \multicolumn{2}{|l|}{ Obesity } \\
\hline $\mathrm{BMI} \geq 25$ & $22(21.8 \%)$ \\
\hline $\mathrm{BMI}<25$ & $79(78.8 \%)$ \\
\hline \multicolumn{2}{|l|}{ Past history of thyroid surgery } \\
\hline Yes & $16(15.8 \%)$ \\
\hline No & $85(84.2 \%)$ \\
\hline \multicolumn{2}{|l|}{ Thyroid pathology } \\
\hline Papillary carcinoma & $92(91 \%)$ \\
\hline Anaplastic carcinoma & $3(3 \%)$ \\
\hline Medullary carcinoma & $2(2 \%)$ \\
\hline Follicular carcinoma & $1(1 \%)$ \\
\hline Benign tumor & $3(3 \%)$ \\
\hline \multicolumn{2}{|l|}{ Surgical procedure } \\
\hline Hemithyroidectomy + central ND & $49(49 \%)$ \\
\hline Total thyroidectomy + central ND & $17(17 \%)$ \\
\hline Total thyroidectomy + bilateral ND & $8(8 \%)$ \\
\hline Total thyroidectomy + unilateral ND & $6(6 \%)$ \\
\hline Hemithyroidectomy + unilateral ND & $5(5 \%)$ \\
\hline ND only (secondary operation) & $16(16 \%)$ \\
\hline
\end{tabular}

Note. SD: standard deviation, ND: neck dissection, BMI: body mass index.

VF mobility could be observed by US evaluation in 89 patients out of the 100 patients whose VFs could be observed by electroendoscopic examination before thyroid surgery. In other words, we had 178 observations of VF mobility combined right and left VFs. After thyroid surgery, 89 out of 101 patients could be observed by US evaluation (178 observations). Sensitivity, specificity, positive and negative predictive values of US evaluation were $87.5 \%, 100 \%, 100 \%$, and $99.4 \%$ before and $94.4 \%, 100 \%, 100 \%$, and $98.6 \%$ after the thyroid surgery (see Table 2 ).

Table 2. Assessment of vocal fold movement (right and left results were combined)

\begin{tabular}{lll}
\hline & Paralysis (+) by EE & Paralysis (-) by EE \\
\hline Before thyroid surgery & & \\
Paralysis (+) by US & 7 & 0 \\
Paralysis (-) by US & 1 & 170 \\
After thyroid surgery & & \\
Paralysis (+) by US & 34 & 0 \\
Paralysis (-) by US & 2 & 142 \\
\hline
\end{tabular}

Note. US: ultrasonography; EE: electroendoscopy. 
Figure 2. The images of ultrasonography with transverse view at the level of the thyroid notch. (A): A case with normal mobility of vocal folds. Bilateral false vocal folds show symmetrical abduction and adduction movements; (B): A paralysis case. The left false vocal fold shows limited abduction movement.

Note. The images of ultrasonography with transverse view at the level of the thyroid notch. The left panel of these pictures shows closing glottis at the time of expiration, and the right panel shows opening glottis at the time of inspiration.
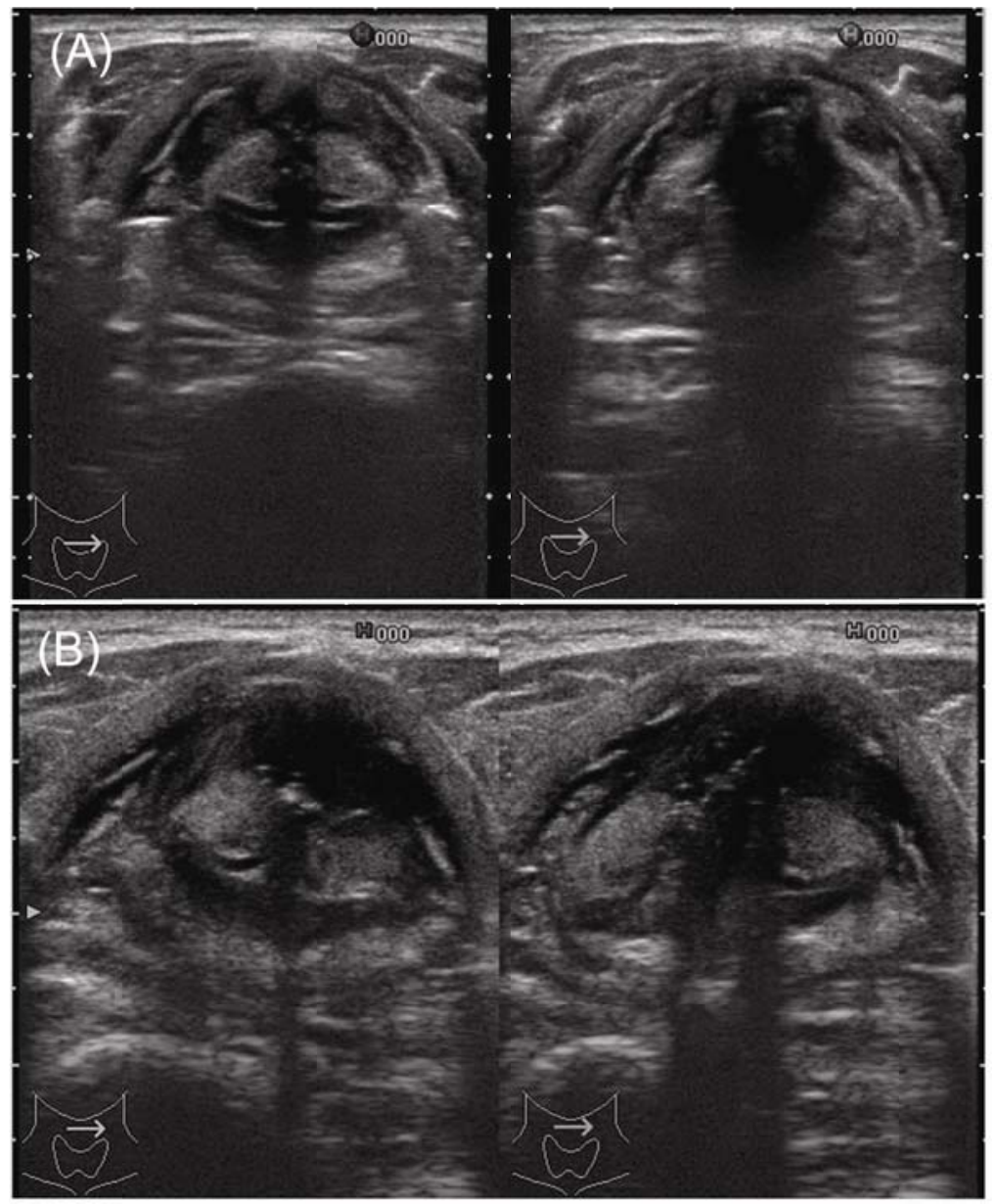

Comparing clinical profiles between patients with visible and invisible VF mobility on US (see Table 3), strong significant differences were observed for men (OR 24.7, 95\% CI 4.9-124.2), 65 years old or more (OR 3.5, 95\% CI 1.0-11.9), and have past history of thyroid surgery (OR 7.9, 95\% CI 2.1-29.2). Although obesity was not a significant factor, stratified analysis identified obesity was an independent predictor (OR 10.5, 95\% CI 1.7-64.8) of invisible on US only among younger patients (less than 65 years old) (see Table 4).

In preoperative examination, the case that could not be visualized by EE was excluded $(n=1)$. US could visualize the vocal fold (VF) mobility in 89 of 100 patients. Sensitivity $=87.5 \%$, specificity $=100 \%$, positive predictive value $=100 \%$, and negative predictive value $=99.4 \%$.

In postoperative examination, VF mobility could be visualized by EE in all of the 101 cases. US could visualize the VF mobility in 89 of 101 patients. Sensitivity $=94.4 \%$, specificity $=100 \%$, positive predictive value $=100 \%$, and negative predictive value $=98.6 \%$.

Table 3. Crude Odds ratios for NOT being able to visualize vocal fold mobility by ultrasonographic evaluation before thyroid surgery

\begin{tabular}{lll}
\hline & Odds ratio & $\mathbf{9 5 \% ~ C I ~}$ \\
\hline Age $(65 \geq$ vs. $65<)$ & 3.5 & $1.0-11.9$ \\
Sex (men vs. women) & 24.7 & $4.9-124.2$ \\
Obesity (BMI $\geq 25$ vs. BMI $<25)$ & 2.0 & $0.5-7.3$ \\
Prior operation $(+$ vs. -$)$ & 7.9 & $2.1-29.2$ \\
\hline
\end{tabular}

Note. OR: odds ratio; $95 \% \mathrm{CI}$ : 95\% confidence interval. 
Table 4. Stratum specific Odds Ratios with obesity for NOT being able to visualize vocal fold mobility by ultrasonographic evaluation before thyroid surgery stratified by sex, age, and prior operation history

\begin{tabular}{lll}
\hline & Odds ratio & $\mathbf{9 5 \%} \mathbf{C I}$ \\
\hline Stratified by sex & 1.6 & $0.19-13.9$ \\
Men & $\mathrm{N} / \mathrm{A}$ & $\mathrm{N} / \mathrm{A}$ \\
Women & & \\
Stratified by age & 10.5 & $1.7-64.8$ \\
Age $\geq 65$ & $\mathrm{~N} / \mathrm{A}$ & $\mathrm{N} / \mathrm{A}$ \\
Age $<65$ & & \\
Stratified by prior operation & 1.2 & $0.1-10.2$ \\
yes & 2.1 & $0.4-12.8$ \\
No & & \\
\hline
\end{tabular}

Note. $95 \%$ CI: $95 \%$ confidence interval; N/A: not applicable.

\section{Discussion}

The present study found that US could correctly assess recurrent laryngeal nerve function both before and after thyroid surgery, and the sensitivity and specificity were considerably high. However, it could not be available in all of the cases (almost $90 \%$ of the study patients).

There were four factors that made it difficult for US to observe VF mobility; men, older age (65 years old or more), past history of thyroid surgery, and obesity among the patients younger than 65 years. First, the difficulty to assess the VF mobility by US in men compared with in women may mainly be due to anatomical differences of the larynx between men and women. In the case of adults, the external angle between the blades of the thyroid cartilage is more acute in men than in women and the distance from the thyroid notch to the anterior commissure is greater in men than in women ${ }^{[12]}$. In other words, it is difficult to appropriately place the flat transducer on the acute angular thyroid cartilage of men, and the distance from the surface of the transducer to the VF tends to be greater in men than in women. Second, the difficulty to assess the VF mobility by US in older patients may mainly be due to increasing thyroid cartilage ossification with advancing age, which processes more extensive in men than in women ${ }^{[13]}$. Third, past history of thyroid surgery may cause postoperative scaring or fibrosis which may make it more difficult for US to observe VF mobility. At last, the neck subcutaneous fat thickness of the patients with obesity may affect the accuracy of US evaluation for VF mobility. The reason why high BMI was not a significant factor among older age group may be due to the fact that BMI is originally an indicator to assess abdominal obesity and obesity will progress with aging mainly at lower parts of the trunk such as the waist and infragluteal region ${ }^{[14]}$. Wang et al. also found that US evaluation of VF mobility was significantly more difficult in men than in women, and in older patients than younger patients, which were compatible with the present study ${ }^{[6]}$.

The use of electrocautery during surgery may cause flap edema and greatly thicken the flap ${ }^{[15]}$. Actually, in the present study, the thickness of the flap measured by the minimum distance from the surface of the neck skin to the thyroid cartilage came to be significantly greater after surgery $(4.9 \pm 0.3 \mathrm{~mm}$, mean, standard error) than before surgery $(3.3 \pm 0.2 \mathrm{~mm}$, $p<.001, \mathrm{n}=82$, data not shown), and actually in one case of which the VFs could have been observed by US before surgery, the VFs came to be invisible by US after the surgery because of severe flap edema. However, validity of US evaluation for VF mobility did not change essentially between before and after surgery.

The thyroid cartilage will provide the best window for US imaging of the VF, and VF will also be well visible through the cricothyroid membrane, while the probability to visualize VF through thyrohyoid membrane is $71 \%{ }^{[16]}$. Observation from all of 3 points (thyroid membrane, thyroid notch, and cricothyroid membrane) may a little improve the accuracy of US evaluation for VF, and in one rare case that electroendoscopic examination could not confirm the exact VF mobility due to 
a protruding metastatic retropharyngeal lymph node over the larynx, the VF mobility could be observed by US. Thus US may also be useful for observation of VF mobility in the case that laryngeal fiber scope is ineffective for observation of the VF.

In Japan, since the charge for laryngeal fiberscopic examination is much more expensive than simple thyroid ultrasonography, repeating both of US and laryngeal fiberscopic examination may be too heavy a burden for the patients. Considering the cost performance and the fact that US evaluation is indispensable to examine thyroid diseases and many patients with thyroid cancer are non-smoking elderly women, laryngeal fiberscopic examination may be able to be omitted from the routine pre- and postoperative examination of thyroid surgery, when US evaluation can properly detect the VF mobility and tumor invasion to the larynx or trachea is not suspected. However, up to $30 \%$ of patients with normal VF mobility after thyroid surgery complain of subjective voice changes of which exact cause remains unclear ${ }^{[17,18]}$. In such patients with postoperative symptoms, fiberscopic examination should be performed to protect the surgeon from subsequent litigation ${ }^{[19]}$ or not to overlook any other abnormalities in the larynx, even when VF mobility can be observed by US.

In the present study, there was one limitation that since only one experienced radiologist performed US, general reproducibility and interobserver variability were uncertain, although the technique to handle US for observation of the VF is not difficult.

\section{Conclusion}

The present study suggests that US evaluation can be used for the assessment of recurrent laryngeal nerve function before and after thyroid surgery. This is a safe and non-invasive diagnostic tool which may be performed as a first-line method. Laryngeal fiberscopic examination should be performed only when US evaluation is ineffective or the patient is symptomatic. Or at least, repeated laryngeal fiberscopic examination may be unnecessary when VF mobility can be evaluated by US.

\section{References}

[1] Sugitani I, Kasai N, Fujimoto Y, et al. A novel classification system for patients with PTC: Addition of the new variables of large ( $3 \mathrm{~cm}$ or greater) nodal metastases and reclassification during the follow-up period. Surgery. 2004; 135: 139-48. http://dx.doi.org/10.1016/S0039-6060(03)00384-2

[2] Bisetti MS, Segala F, Zappia F, et al. Non-invasive assessment of benign vocal folds lesions in children by means of ultrasonography. Int J Pediatr Otorhinolaryngol. 2009; 73(8): 1160-2. PMid:19497627 http://dx.doi.org/10.1016/j.ijporl.2009.05.004

[3] Ko HC, Lee LA, Li HY, et al. Etiologic features in patients with unilateral vocal fold paralysis in Taiwan. Chang Gung Med J. 2009; 32(3): 290-6. PMid:19527608

[4] Dedecjus M, Adamczewski Z, Brzeziński J, et al. Real-time, high-resolution ultrasonography of the vocal folds-a prospective pilot study in patients before and after thyroidectomy. Langenbecks Arch Surg. 2010; 395(7): 859-64. PMid:20640934 http://dx.doi.org/10.1007/s00423-010-0694-2

[5] Sulica L, Blitzer A. Vocal fold paresis: evidence and controversies. Curr Opin Otolaryngol Head Neck Surg. 2007 ; $15(3)$ : $159-62$. PMid:17483683 http://dx.doi.org/10.1097/MOO.0b013e32814b0875

[6] Wang CP, Chen TC, Yang TL, et al. Transcutaneous ultrasound for evaluation of vocal fold movement in patients with thyroid disease. Eur J Radiol. 2012; 81(3): 288-91. PMid:22019598 http://dx.doi.org/10.1016/j.ejrad.2011.09.020

[7] Farrag TY, Samlan RA, Lin FR, et al. The utility of evaluating true vocal fold motion before thyroid surgery. Laryngoscope. 2006; 116: 235-8. PMid:16467711 http://dx.doi.org/10.1097/01.mlg.0000191472.02720.1f

[8] Vats A, Worley GA, de Bruyn R, et al. Laryngeal ultrasound to assess vocal fold paralysis in children. J Laryngeal Otol. 2004; 118(6): 429-31. http://dx.doi.org/10.1258/002221504323219545 
[9] Wang CP, Chen TC, Lou PJ, et al. Neck ultrasonography for the evaluation of the etiology of adult unilateral vocal fold paralysis. Head Neck. 2012; 34(5): 643-8. PMid:21692133 http://dx.doi.org/10.1002/hed.21794

[10] Cheng SP, Lee JJ, Liu TP, et al. Preoperative ultrasonography assessment of vocal cord movement during thyroid and parathyroid surgery. World J Surg. 2012; 36(10): 2509-15. PMid:22689020 http://dx.doi.org/10.1007/s00268-012-1674-1

[11] Ng SK, Yuen HY, van Hasselt CA, et al. Combined ultrasound/endoscopy-assisted vocal fold injection for unilateral vocal cord paralysis: a case series. Eur Radiol. 2012; 22: 110-3. PMid:22130628 http://dx.doi.org/10.1007/s00330-011-2337-6

[12] Jotz GP, Stefani MA, da Costa Filho OP, et al. A morphometric study of the larynx. J Voice. 2014; 28(6): 668-72. PMid:24814367 http://dx.doi.org/10.1016/j.jvoice.2014.03.008

[13] Dang-Tran KD, Dedouit F, Joffre F, et al. Thyroid cartilage ossification and multislice computed tomography examination: a useful tool for age assessment? J Forensic Sci. 2010; 55: 677-83. PMid:20345806 http://dx.doi.org/10.1111/j.1556-4029.2010.01318.x

[14] Murakami M, Hikima R, Arai S, et al. Short-term longitudinal changes in subcutaneous fat distribution and body size among Japanese women in the third decade of life. Appl Human Sci. 1999; 18: 141-9. PMid:10510517 http://dx.doi.org/10.2114/jpa.18.141

[15] Uludag M, Yetkin G, Ozel A, et al. Wound complications and clinical results of electrocautery versus a scalpel to create a cutaneous flap in thyroidectomy: A prospective randomized trial. Surg Today. 2011; 41: 1041-8. PMid:21773891 http://dx.doi.org/10.1007/s00595-010-4435-5

[16] Singh M, Chin KJ, Chan VW, et al. Use of sonography for airway assessment: an observational study. J Ultrasound Med. 2010; 29: 79-85. PMid:20040778

[17] de Pedro Netto I, Fae A, Vartanian JG, et al. Voice and vocal self-assessment after thyroidectomy. Head Neck. 2006; $28: 1106-14$. PMid:16933312 http://dx.doi.org/10.1002/hed.20480

[18] Stojadinovic A, Shaha AR, Orlikoff RF, et al. Prospective functional voice assessment in patients undergoing thyroid surgery. Ann Surg. 2002; 236: 823-32. PMid:12454521 http://dx.doi.org/10.1097/00000658-200212000-00015

[19] Schlosser K, Zeuner M, Wagner M, et al. Laryngoscopy in thyroid surgery-essential standard or unnecessary routine? Surgery. 2007; 142: 858-64. PMid:18063068 http://dx.doi.org/10.1016/j.surg.2007.09.008 